\title{
Efficient data sensing and monitoring model for areca nut precision farming with wireless sensor network
}

\author{
Niranjan Murthy Chandrashekarappa ${ }^{1}$, SanjayPande Mysore Bhagwan ${ }^{2}$, \\ Kotreshi Shivabasappa Nagur ${ }^{2}$ \\ ${ }^{1}$ Department of Computer Scince and Engineering, Visweswaraya Technological University, Belgavi, India \\ ${ }^{2}$ Department of Computer Scince and Engineering, Gowdar Mallikarjunappa Institute of Technology, Davanagere, India
}

\begin{tabular}{l} 
Article Info \\
\hline Article history: \\
Received Jul 14, 2021 \\
Revised Dec 24, 2021 \\
Accepted Jan 13, 2022 \\
\hline
\end{tabular}

Keywords:

Efficient data sensing and monitoring

Energy consumption

NPK

Temperarture

Wireless sensor networks

\begin{abstract}
Areca nut plays a prominent role in economic life in India; it produces 'betel nut' which is primarily used for the masticatory purpose. Nutrient's cycle and environmental factors impact the forming, these impacts can be minimized through sensing technology i.e., wireless sensor network incorporated with internet of things (IoT). Designing of sensing technologies is considered as primary steps in achieving the arecanut production through precision agriculture; This research focuses on designing and developing an efficient monitoring mechanism named efficient data sensing and monitoring (EDSM), the proposed model will minimize the energy, reduce the false alarm rate, and enhance the detection accuracy. EDSM comprises four-step optimal sensing mechanism; first, formulate the energy consumption, further in this step the sensor device information and all the preliminary details are analyzed. Second step, data are sensed optimally, third step includes monitored and alert is generated the fourth step includes the optimization of packet size. EDSM is evaluated considering the different parameters like energy consumption and alert generation for temperature. Performance comparison is carried out with the existing model considering parameters like fault detection, false alarm detection, event detection, and event false alarm rate. Comparative analysis shows proposed methodology simply outperforms the existing model with significant improvisation.
\end{abstract}

This is an open access article under the CC BY-SA license.

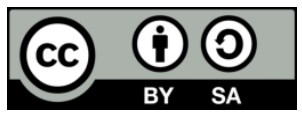

\section{Corresponding Author:}

Niranjan Murthy Chandrashekarappa

Department of Computer Scince and Engineering, Visweswaraya Technological University

Belgavi, India

Email: nirucs190@gmail.com

\section{INTRODUCTION}

The essential resources for humanity's survival and progress are food, water, and energy. The worldwide demand for these resources has been steadily rising, and this trend is projected to continue. According to the United Nations, the world's population will reach 9.8 billion by 2050 and 11.2 billion by 2100 [1]. With the influence on, the environment the occurrence of global warming, major crop-producing countries achieving their freshwater limits, unpredictable climate variability, decreased land fertility due to drought, erosion, and poor management, and the ever-increasing public aspirations for more feasible practices to decrease water and agrochemical use, in agriculture, efficiency and sustainability has never been more crucial [2].

Nutrient cycling has happened naturally throughout the bulk of Earth's history, from soil to plants and animals, and then back to the soil through biomass breakdown. The natural soil nutrient cycle was disrupted when people progressed from hunter-gatherers to cultivating agriculture and establishing long-term 
settlements, thanks to the manufacturing and application of agrochemicals. The green revolution, which began in the mid-twentieth century and expanded agricultural output globally via selective breeding for producing and adopting high-yielding crop varieties, particularly cereals, and innovative farming approaches, further altered agriculture. Since then, humanity's objective has been to achieve sustainability through precision agriculture. In general, 17 key elements/nutrients have been identified as being vital for plant growth, and a lack of any one of them might result in lower yields [3]-[5].

The common chewing nut, also known as betel nut or supari, is produced by the areca nut palm, since this nut is widely consumed, it is in high demand areca nut is closely associated with religious traditions in India. India is the world's top producer and consumer of areca nuts; areca nut is a fruit that is classified as a drupe rather than a real nut. Dried, cured, and fresh versions are commercially available. In India, commercial cultivation of the areca nut has been more successful. This nut palm falls to the "Arecaceae" class and the "Areca L" genus. Kerala, Meghalaya Assam, Tamil Nadu, and Karnatakaare among the states that cultivate the Arecanutcrop. Arecanuts may be cultivated in a variety of soil types. This crop, on the other hand, does best on well-drained soils with plenty of organic matter. To avoid sunburn, proper shielding from the sun's rays from the west is required. Before planting arecanut seedlings, fast-growing shade trees should be planted on the southern and western sides. This palm nut tree is susceptible to water shortages and should only be cultivated in areas with adequate irrigation. A well-distributed yearly rainfall of 750 to $4500 \mathrm{~mm}$ is required for this crop. This crop may be produced at elevations as high as 1000 meters above sea level (MSL). The recommended temperature range for its growth and yield is $10^{\circ} \mathrm{C}$ to $40{ }^{\circ} \mathrm{C}$ [6].

Wireless sensor networks (WSN) are a common use of information technology in agriculture, capable of monitoring soil information in the regions of interest and encouraging the traditional irrigation system. By gathering data during the agricultural production process, it can improve irrigation methods and make agriculture smarter [7]. WSNs are critical in the notion of smart agriculture since they monitor and gather interest data in agricultural fields for use in numerous applications. Smart agriculture makes significant efforts to realize precision irrigation, fertilizers, and pesticides based on the crop development model and WSNs in agriculture to reduce water waste, low soil fertility, fertilizer abuse, and illnesses. Sensor nodes in WSNs planted in fields are capable of regularly relaying gathered soil information data to the sink node through wireless communication [8]. WSNs are utilized to capture temperature, humidity, soil moisture content, and wind speed data in an agricultural application, according to the authors. For crops, a WSNsbased IoT irrigation control system is suggested to manage the moisture content of the soil for smart farms, which may successfully cut costs and boost agricultural output [9]. Figure 1 shows the typical architecture of WSN based agriculture architecture where the data are sensed at the ground and through the internet, it is sent to the internet cloud where the data can be read and analyzed through an application.

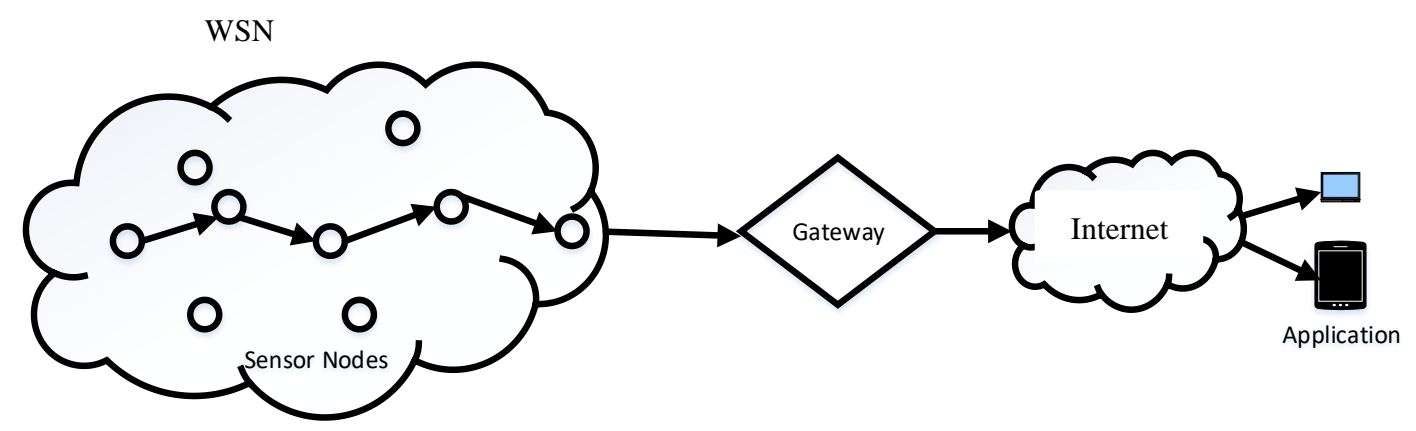

Figure 1. Typical WSN architecture for precision farming

WSNs are constantly in danger of faults and failures due to their resource-constrained nature and unique properties. The reason for this is that the gathering and sending of data consume the majority of the sensor network's energy. As a result, the majority of the solutions proposed centered on energy-aware computing with dispersed data collecting. Clustering is another extensively used energy reservation strategy in WSNs. Each zone chooses a master node (cluster head $(\mathrm{CH})$ ) to gather data from all nodes and transfer it to the BS. CH only communicates with the BS-managed network topology, which saves a lot of energy. Sensor nodes in WSNs typically operate in a continuous sensing and processing mode. It also conducts data aggregation and forwarding of detected data, lowering node energy consumption, and extending network life. Numerous techniques have been developed to reduce energy usage and extend network life. The majority of 
these approaches have primarily focused on energy dissipation. Various protocols provide different steps for controlling and monitoring network operations [10], [11].

A. Motivation and contribution of research work

Farming is considered as a foundation of any national as well as harmonious society and for the past several years' government has been focusing on precision farming; moreover, there are various types of nuts produced in India, areca nut is one of the largest productivities in our country. Optimum availability of nutrients is essential for the different biophysical processes such as plant growth, seeds germination, nutrient cycle and also to sustain the soil biodiversity; also, to achieve the precision farming environmental factor like temperature and humidity needs to not only sensed but monitor and generate the alarm as well. Thus, motivated by the application and commercial aspect, this research work focuses non designing the energy aware EDSM model; further contribution of the research work is highlighted through the points: i) We design and develop efficient data sensing and monitoring (EDSM) model to achieve the precision farming of areca nut; ii) EDSM comprises four distinctivesteps; the first step includes the gathering information about the sensors, energy, and other preliminaries, the second step involves the sensing of data, the third step involves the monitoring and alert generation, fourth and last step includes the optimization of packet size along with monitoring and alert generation; iii) EDSM minimizes energy consumption and monitors essential factors such as temperature, humidity, and NPK value; and iv) Comparative analysis is carried out to prove the model efficiency and EDSM outperforms the existing model with significant improvisation.

This research work is organized in such a way that the section 1 starts with the background and importance of precision farming; further in the same section importance of areca nut farming and the requirement for precision farming is discussed. Furthermore, sensing technology based on WSN isdiscussedandthe section 1 ends with the motivation and contribution of the research article. In the section 2, several related works for precision farming along with sensing technologies are reviewed and shortcomings of the same are highlighted. The section 3 presents the mathematical modeling of the proposed methodology along with the algorithm; the further model is evaluated in the section 4 along with a performance comparison with an existing model.

\section{RELATED WORK}

This section reviews the various efficient sensing mechanism developed; in general, most of the methods had an approach of clustering and routing; the most well-known clustering routing mechanism for WSNs is low-energy adaptive clustering hierarchy (LEACH) [12]. In LEACH, the $\mathrm{CH}$ is chosen depending on the current round's likelihood; as a result, $\mathrm{CH}$ selection is not evenly spread, resulting in unconnected nodes. Multihop-LEACH [13] is a modification of the LEACH protocol that allows for multi-hop intercluster and intra-cluster communication. Sensors collect data and communicate it to $\mathrm{CHs}$ based on the timeout duration; forwarding vexing messages can lead to a never-ending use of electricity. Another modified type of LEACH is the Quadrature-LEACH (Q-LEACH) [14]; Q-LEACH has been enhanced for both power management and improved service, nonetheless, all LEACH modifications are based on hierarchical clustering, but hierarchy is not the best approach in WSNs. For a better selection of CH based on remaining energy, hybrid LEACH [15], [16] is employed where $\mathrm{CH}$ is chosen between high and low energy levels using threshold conditions for each iteration. PEGASIS [17] has eliminated all of LEACH's flaws. For a leader to send data to BS, just two messages will be necessary instead of the twenty messages necessary in LEACH for complete coverage. However, under this method, some superfluous and bulky messages circulate in the network, resulting in node energy levels being reduced. CEER [18] is another multi-hop communication architecture that may be used for both CHs and BS. This method runs in rounds, with each node calculating its fitness function based on distance, residual energy, and probability. Based on the Genetic algorithm, BS chooses $\mathrm{CH}$. This technique, on the other hand, fails to manage the algorithm and choose the optimum path. SEP in [19] has been developed to balance energy usage in fog supported WSNs. CHSis chosen depending on the remaining energy and distances traveled using CHs. For the main and secondary $\mathrm{CH}$ election processes, a threshold is defined for verifying energy and other characteristics. SEP's successor is the prolong SEP [20] protocol. On the GPS interface, a location-based protocol such as the MECN [21] is used to compute sub-networks with the least amount of energy. When the network is dispersed and impediments arise between neighboring nodes, failure happens. Another location-based protocol is global assessment of functioning (GAF) [22], which was created for Mobile Ad hoc networks and then updated for WSNs. Nodes of the virtual grid communicate with one another in predefined zones. When SN mobility is combined with dynamically changing topologies, the system appears to be unreliable. Geographic and energy aware routing (GEAR) [23] generates geographic data, which is then utilized to pick CH. GEAR is useful for network management in general, but it is not suggested for low-energy communication networks such as WSNs. SPIN [24] is a data-centric energy conservation technique in where each node acts as a BS. Duplicate data packets are deleted based on routing negotiation. A negotiation message, on the other hand, causes 
network congestion. COUGAR [25], a data-centric strategy that organizes the network in a distributed database system, is another version. Abstract query processing is used at the network layer for data collecting, aggregation, and forwarding. WSNs require lower layer rules for aggregation and data forwarding, but the COUGAR operates on the higher layer where more energy is gathered. Other systems provide numerous parameters for balanced $\mathrm{CH}$ selection and similar clusters, including energy, distance [24], coverage, and convergence regions [26]. A delay and energy aware coordination protocol (DEACP) in [16] has been suggested as an energy-efficient routing protocol. Dropping chance is reduced when all cluster members use a queue overflown routing scheme. It controls the dissipation of energy across all nodes. The $\mathrm{CH}$ selection has been improved, and the entire region is covered with complete connection and little energy use. According to a sleeping schedule, a node's radio module is switched off for a specific amount of time [27]. Presents a full study of routing in WSNs and IoT.

Wan et al. [28] presented the recent development observed in the IoT domain which mainly focused on the various sensing mechanism some of them focused on clustering and some of them were cluster head based. Another state-based system is the energy-efficient sleep scheduling mechanism with similarity measure (ESSM) [29]. The ESSM method is based on switching a node's status from active to sleep. The algorithm has been designed with condensed sensor deployment in mind, making it suitable for crowded WSNs. However, with a distributed deployment, data correlation will be low, and the network would consume more energy. A state-based technique for underwater acoustic sensor networks is an effective scheduling algorithm for coverage control in underwater acoustic sensor networks (UASNs) [30]. The ESACC active-sleep approach is implemented in two halves, each with redundant nodes. For sense, a memetic technique is employed to activate the sleep state, while certain nodes will be in an active state. During network activities, the drowsy nodes are awakened and become Active/Live. The introduction of a harvesting layer in WSNs has been proposed in A method of balanced sleep scheduling in renewable wireless sensor networks (BSSR) [31]. Moreover, few recent approaches [32]-[34] follows the dynamic approach of the sensing mechanism in the different area of agriculture such as water management, location-based longterm monitoring; these approach does provide the solution to any problem in agriculture. However, it is observed through the related work that all these mechanisms either focus on a single scenario or they are not efficient enough to be deployed in real-time.

\section{PROPOSED METHOD}

Optimum availability of nutrients is essential for the different biophysical processes such as plant growth, seeds germination, and nutrient cycle and also to sustain the soil biodiversity; also, to achieve the precision farming environmental factor like temperature and humidity needs to not only sensed but monitor and generate the alarm as well. Thus, considering the above factor, in this section, we design and develop a mathematical model of the efficient data sensing and monitoring (EDSM) mechanism; EDSM not only senses the data efficiently but also monitors it and generates alerts if it exceeds. Moreover, EDSM comprises several parts which are depicted in Figure 2.

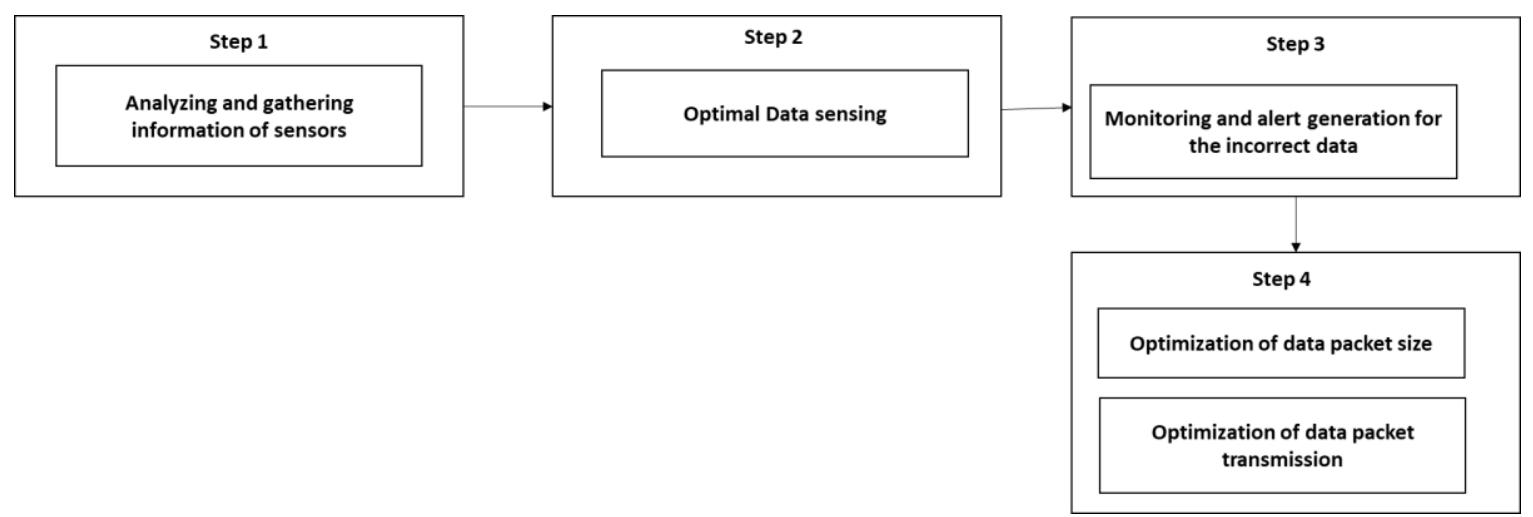

Figure 2. Efficient data sensing and monitoring mechanism

\subsection{Energy consumption}

Let's consider a sensory field that has limited energy since it is considered battery-based, thus the network lifetime in terms of energy can be given as: 


$$
M=\left(\text { energy }_{\text {bit }}\right)\left(C_{\max }\right)
$$

furthermore, the issue of data gathering can be defined as (2):

$$
g_{d}\left(S, \text { energy } y_{\text {total }}\right) \leq M
$$

where $S=\left\{s_{1}, s_{2} \ldots s_{O}\right\}$ and $d=1,2, \ldots \ldots, D$; in above two equation $C_{\max }$ is maximum threshold bit that is transmitted or received in a given certain time, energy $y_{b i t}$ indicates the energy cost to transmit or receive the single-bit data and $S$ indicates the measure data with $D$ indicating total constraints and $O$ as the number of samples. The (3) as (3):

$$
\text { energy }_{\text {total }}=\text { energy }_{\text {total }}(w, v, e)
$$

with subjected to:

$$
\operatorname{minimize}\{\{g(w), g(v), g(e)\}\}
$$

$g(v)$ indicates the optimizing the updating time while collecting the data, $g(w)$ indicates the function to optimize the data validation and avoiding the missense data and $g(e)$ indicates the optimization function of the transmission issue. Furthermore, through the analysis of a various existing model of smart farming, it was observed that faulty data transmission leads to high energy consumption and avoidance of same can result in optimization of energy, also it was noted that faulty data detection and transmission can be computed invalidation step and can be computed through equation.

$$
\begin{aligned}
& \text { Energy }_{\text {sec Phase }}\left(Q_{\text {time }}\right)=\text { Energy }_{\text {read }}\left(P_{\text {time }}\right)+\text { Energy }_{\text {Transmit }}\left(Q_{\text {time }}\right)+ \\
& \text { Energy }_{\text {adjneighbour }}\left(Q_{\text {time }}\right)
\end{aligned}
$$

In the equation, Energy $y_{\text {adjneighbour }}\left(Q_{\text {time }}\right)$ indicates the energy required to gather data from

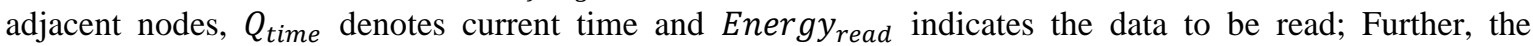
equation can be re-written as (5):

$$
\begin{aligned}
& \operatorname{Energy}_{\text {secPhase }}\left(Q_{\text {time }}\right)=\sum_{Q_{\text {time }}=1}^{O Q}\left(\text { Energy }_{S D}\left(Q_{\text {time }}\right)+\left(I_{\text {dsbits }} \times \text { Energy }_{\text {Transmit }}\right)+\right. \\
& \text { Energy } \left._{\text {adjneighbour }}\left(Q_{\text {time }}\right)\right)
\end{aligned}
$$

$I_{d s b i t s}$ is the data size of the transmitted packet; Further, the energy required to receive the data from the

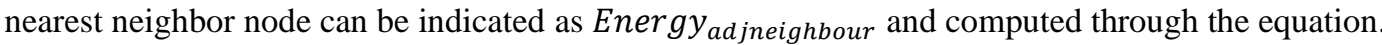

$$
\text { Energy } y_{\text {adjneighbour }}\left(Q_{\text {time }}\right)=\sum_{j=1}^{O_{\text {adjacentnode }}}\left(\text { Energy }_{\text {received }} \times I_{R d s \text { bits }}\right)
$$

$I_{R d s ~ b i t s}$ indicates received data size and Energy $y_{\text {received }}$ is a parameter for the energy required to receive the unit bit and $O_{\text {adjacentnode }}$ indicates nearest neighbor nodes.

$$
\text { Energy }_{\text {read }}=\left(\sum_{j=1}^{I} S_{\text {bits }} \times S_{\text {Ecost }}\right)+\left(\sum_{j=1}^{I} X_{\text {bits }} \times X_{B \text { cost }}\right)
$$

In the equations, $S_{\text {bits }}$ and $X_{\text {bits }}$ indicates the number of reads and write bits; $S_{E c o s t}$ and $X_{B c o s t}$ indicates the energy required to read and write the data. Further, energy consumption while optimization step is denoted as $\varepsilon_{R D-P h a s e}$ and formulated through the equation where $I_{1}, I_{2}$ and $I_{3}$ are considered as the forwarded sample data.

$$
\begin{aligned}
& \text { Energy } y_{\text {thirdPhase }}=\left(\sum_{j=1}^{I_{1}} \operatorname{Energ}_{\text {param } 1(j)}\right)+\left(\sum_{j=1}^{I_{2}} \operatorname{Energ}_{\text {param } 2(j)}\right)+ \\
& \left(\sum_{j=1}^{I_{3}} \operatorname{Energ}_{\text {param } 3(j)}\right)
\end{aligned}
$$

In the equation, Energy $y_{\text {param } 3}$ is (9):

$$
\text { Energy }_{\text {param } 1}=\left(\text { basic }_{\text {length }} \times \text { Energy }_{\text {Trans }}\right)
$$


where param 2 indicates the data sent from sensor node to the cluster head; thus energy consumption for the param2 is computed as (10):

$$
\text { Energy }_{\text {param } 2}=\left(\text { Energy }_{\text {param } 1} \times\left(1-\frac{\text { optimal }_{\text {length }}}{\text { basic }_{\text {length }}}\right)\right)
$$

in the equation, optimal length and basic $_{\text {length }}$ indicates the length parameter. Further energy consumption for param3is given as (11):

$$
\text { Energy }_{\text {param } 3}=\text { Energy }_{\text {param } 2}+\left(\text { refparam }_{\text {length }} \times \text { Energy }_{\text {Trans }}\right)
$$

total energy consumption is (12).

$$
\text { Energy }_{\text {Total }}=\text { Energy }_{\text {fourthPhase }}+\text { Energy }_{\text {thirdPhase }}
$$

\subsection{Efficient data sensing and monitoring}

In this section, we develop efficient real-time data collection; optimal data collection comprises different steps; the first step includes the sensing device condition, the second step includes updating the data strategy, the third step includes data validation, and the fourth step includes the sensed data optimizationThe term sensing device refers to a device that collects data from various sensors and calculates how much energy is consumed while sensing and sending data. Later updates imply that data is returned to the user for further analysis, that the data is then verified to see if it is valid or incorrect, and that the next procedure is to receive ideal data that is simple to verify and update. In different stages of obtaining data, all possible steps are shown below.

\subsubsection{Obtaining the sensor node information}

This Algorithm 1 deals mainly with the physical sensing device condition as sensed data quality in this step, at first, the prediction model is formulated and computed and a threshold is defined to check the data validation while collecting the data, later single data are transmitted. Threshold prediction algorithm:

Algorithm 1. Algorithm to gathering information regarding the sensor input $[U] T_{x \times o} \in S^{x \times o}, q, x, o$

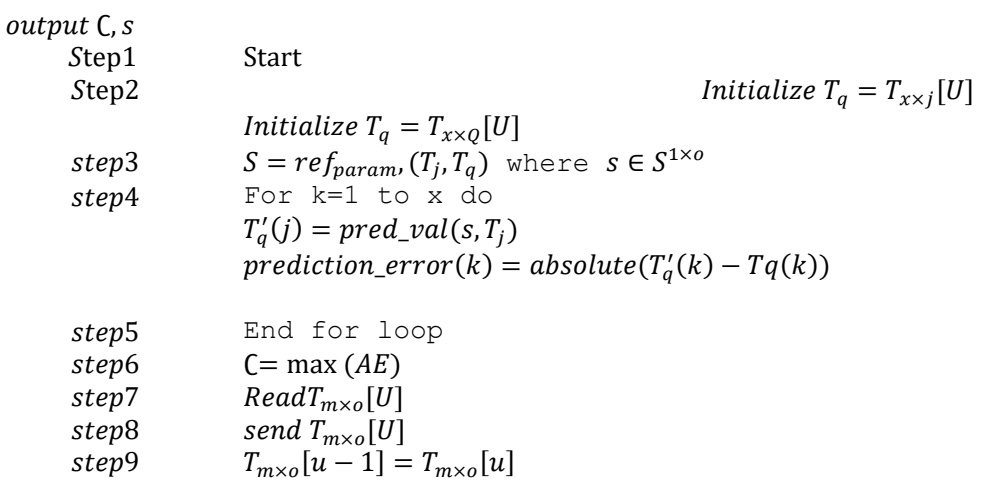

prediction model reference is computed through the (13).

$$
s=\left(T_{j}^{U} \times T_{j}\right)^{-1} \times T_{q} \times T_{j}^{U}
$$

In the equation $T_{q}$ indicates the dependence sensor and $T j$ indicates the independent sensor where $T_{q}$ is computed through $T_{q}=T_{x \times Q}[U]$ and other sensors are indicated as $T_{x \times j}[U]$ where $j=$ 1 to $o$, for all $T q, T j$ belongs to $S^{x \times 1}$. Predicted sensor value function is denoted through $T_{Q}^{\prime}(k)$ and formulated through the (14):

$$
T_{q}^{\prime}(k)=s_{o}+s_{j} \times T_{j}(k)+\cdots s_{o} \times T_{o}(k)
$$


where $j=1$ to $o$ and $k=1$ to $w$ which further indicates collected samples. Furthermore, to compute the threshold, error among the given and predicted data is computed, a further threshold value is computed through choosing the maximum value of approximation error for the given value of $T_{q}$ and error is formulated as (15).

$$
\operatorname{abs\_ } \operatorname{error}(k)=\left|T_{q}^{\prime}(k)-T_{q}^{\prime}(k)\right|, E r \in S^{1 \times x}
$$

\subsubsection{Efficient data sensing and monitoring}

In this sub-step Algorithm 2, data are efficiently sensed; further, this particular step also looks for the device condition in terms of energy, measuring capacity to avoid the inaccurate data sensing; moreover, sensing capacity is computed; sensing capacity defined as the measure of the acceptance of readings achieved through:

$$
\tau=\left(1-\frac{F}{(F+D}\right) \times 100
$$

in the equation, $F$ indicates the detected error and $D$ indicates the correctly measured data (16) computes the network reliability:

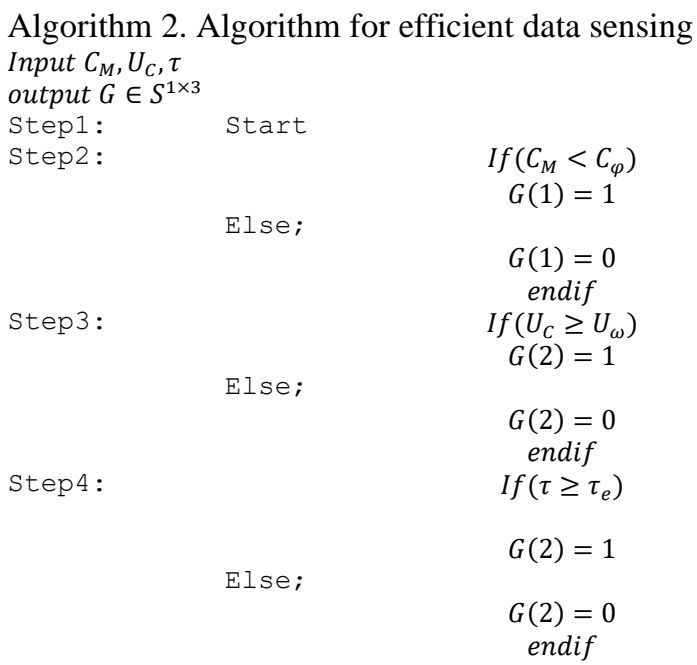

to avoid the transmission of incorrect data and enhance the detection accuracy; we introduce several parameters which contribute to the event detection and detection accuracy. Moreover, the error lies because of various errors discussed below.

\subsubsection{Monitoring and alert generation}

In this step, the detection error rate is computed and further monitored, and an alert is generated if found any faulty data.

Alert Generation: In Algorithm 3, this type of error, the alert is generated if the range exceeds; for instance, in this case, the temperature value ranges from 25 to 30 ; hence if it exceeds then it generates the alert, it is denoted as $\mathbb{X}$.

- $\quad$ Type 1 error: In this type of error, although there is a false alarm presented as true and denoted as $\mathbb{Y}$

- $\quad$ Type 2 error: In this type of error, although the model detects the correctly it is detected as faulty and denoted as $\mathbb{Z}$

Moreover, in our case, we consider the $T_{q}(u)$ as a faulty if it is more than the threshold value, otherwise it is considered as the normal.

\subsection{Optimization of packet transmission}

The main aim of packet transmission optimization Algorithm 4 is to optimize the energy; to achieve the optimization, we introduce a parameter function denoted as paramfunc which computes the relative difference among the currently sensed data and last data transmitted:

$$
\text { paramfunc }=\left[\left(T_{j}[u]-T_{j}[u-1]\right)\left(T_{j}[u]+T_{j}[u-1] * 0.5\right)^{-1}\right]
$$


where $j=1,2 \ldots, o$ and $o$ indicates the total number of sensors. Moreover, recent sensed data is denoted as $\widehat{T}_{m \times o}[u]$ and last data transmitted is denoted as $\widehat{T}_{m \times o}[u-1]$ and packet transmission is carried out through the algorithm, if the sensed data remains the same then proceed, else check the validation in the last step.

Algorithm 3. Algorithm for optimal data motoring and detection error rate computation Input $T_{q}(u), x, C, D$ output Error_rate, $\vartheta$

Step1: Start

Step2:

$$
\begin{gathered}
\operatorname{IF}\left(T_{q}(u)>\operatorname{MaxT}_{q}(u)<\operatorname{MinW}\right) \\
\mathbb{X}=1
\end{gathered}
$$$$
\text { for } j=1 \text { to } x \text { do }
$$$$
e(j)=\operatorname{absolute}\left(T_{q}(u)-C(j)\right)
$$

Step3:

$$
\text { End for }
$$

Step4

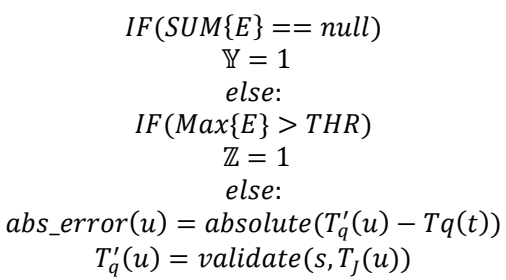

$$
\begin{gathered}
I F((\text { abs_error }(v)>C)) \\
\text { updation in algorithm } \\
T_{q}^{\prime}(u)=\text { validate }\left(s, T_{j}(u)\right) \\
\text { abs_error }(u)=\operatorname{absolute}\left(T_{q}^{\prime}(u)-T q(u)\right)
\end{gathered}
$$

Step5:

$$
\begin{gathered}
\text { If }(\text { abs_error }(u)>C) \\
\vartheta=1 \\
\text { endif }
\end{gathered}
$$

Endif(step4)

Endif(step3)

Endif (step3)

Endif(step2)

Step6:

$\operatorname{Err}=\operatorname{SUM}(\mathbb{X}, \mathbb{Y}, \mathbb{Z})$

Algorithm 4. Algorithm for optimizing the data transmission

Input $o, \tau$

output $E[u]$

Step1:

Step2: Compute maximum number of bits using (8)

Step3: $\quad$ Compute relative difference using (9)

Step 4:

$$
\begin{gathered}
\text { For } \mathrm{j}=1 \text { to o do } \\
\text { If }(\tau(i) \geq 0) \\
\operatorname{nprm}(i)=2^{((j \times M)-1)} \\
\text { else: } \\
n p r m(j)=0 \\
\text { end if } \\
W[j]=A B S\left(\tau(j) \times 2^{((j \times m)-1)}\right) \\
\text { end } \text { for }
\end{gathered}
$$

Step5:

Step 6:

Step 7:

Step8:

$$
\begin{gathered}
\text { compute } E[u]=\operatorname{SUM}[Y] \\
\text { send } E[u] \text { to cluster head } \\
\text { compute } \widehat{T}_{m \times o}[u]=\operatorname{approx}\left(R D, \quad T_{m \times o}[u-1]\right) \\
\text { compute } T_{m \times o}[u-1]=\widehat{T}_{m \times o}[u]
\end{gathered}
$$

\subsection{Optimization of packet size}

In this step, the main intention is to minimize the size of the data packets which further optimizes the energy and sensing vulnerabilities; moreover, in order to optimize the packets a parameter denoted as $\left|\tau_{j}\right|$ which represents the number of bits required for transmission; thus, we compute the maximum number of bits through the (18).

$$
n=\left[\log 2\left(\operatorname{Max}\left(\tau_{1 \times 0}\right)\right)\right]+1
$$


Further, we compute the relative difference through the (19):

$$
M=n+1
$$

considering the above relative difference, positive and negative can be managed through (20).

$$
\text { nprm }_{j}=\left[\begin{array}{l}
0 \quad \text { in case of negative change } \\
2((j \times M)-1) \text { for positive change }
\end{array}\right.
$$

Furthermore, sensed data is represented through the $E[u]$ and formulated through the (21).

$$
E[u]=\sum_{j=1}^{o} \operatorname{nprm}_{j}+\left|\tau_{j}\right| \times 2^{((j \times M)-1)}
$$

Further, we compute the approximated data through the (22) at given time $u$.

$$
\widehat{T}_{m \times o}[u]=T_{m \times o}[u-1]+\left(\left(\tau_{m \times o} \times 10^{-2}\right) \times \widehat{T}_{m \times o}[u-1]\right)
$$

Moreover, the above-presented algorithm is for the end-to-end implementation of EDSM which is carried into different stages. Once the sensing mechanism is designed, evaluation is carried out in the next section of the research.

\section{PERFORMANCE EVALUATION}

In general cost of areca nut cultivation does not make lucrative proposition; however smart farming can lead the industry to be profitable and quality-based venture; thus, this research work proposes EDSM mechanism which efficiently sense the data and monitors for the false data generation. Moreover, EDSM is designed using the sharp as a programming language on programming tool of visual studio 2017; further the system configuration includes the $8 \mathrm{~GB}$ of RAM packed with 2 GB of Nvidiagraphics on windows 10 as operating system. EDSM is simulated through sensoria simulator, further details of proposed model are given through Table 1.

Table 1. System parameter

\begin{tabular}{lc}
\hline \multicolumn{1}{c}{ System parameter } & Value \\
\hline Sensor node considered & 100 \\
Sensor field size & $50 \times 50 \mathrm{~m}$ \\
Sensors & Temperature \\
Initial Energy & $0.05 \mathrm{j}$ \\
Transmission range & $15 \mathrm{~m}$ \\
Sensing range & $8 \mathrm{~m}$ \\
Node failure probability & $0.1-0.5$ \\
\hline
\end{tabular}

\subsection{Alert generation}

At first, we collect the data i.e., raw data of temperature and set the minimum temperature as 25 and maximum temperature as 30 which is ideal for the areca nut; further the data processed is depicted in the below figure. In Figure 3, y-axis presents the temperature and $\mathrm{x}$-axis presents the number of rounds over the given time; moreover, in graph we observe some low and high temperature which suggests that whenever temperature exceeds maximum value of 30 then it generates the alert and further suitable steps are taken. Consider the graph below, which has an $\mathrm{x}$-axis and a $\mathrm{y}$-axis. The temperature range is represented on the $\mathrm{x}$ axis, and the number of nodes deemed to sense data from different nodes is represented on the y-axis, because not all nodes detect correctly. In this graph, the best data is taken into account in order to portray the right numbers.

\subsection{Energy consumption}

WSNs possesses unique characteristics, and it is resource constraints, thus energy consumption plays major role in model efficiency; Figure 4 shows the average energy consumption at different sensor node failure probability; in here we observe that as the sensor failure probability increases average energy consumption also increases such as for 0.1 sensor failure probability, average energy consumption is 0.0267 ; for 0.2 sensor failure probability average energy consumption is 0.0273 . Similarly, for 0.3 and 0.4 sensor failure probability average energy consumptions are 0.0286 and 0.0293 respectively. Furthermore, it should 
be noted that energy consumption is in micro joule. The major goal of this stage is to examine energy usage at various levels of sensor node probability, with 0.1 to 0.4 sensor node probability being used in this study. When transferring data to all sensor nodes in the graph, my methodoloy considers very low energy.

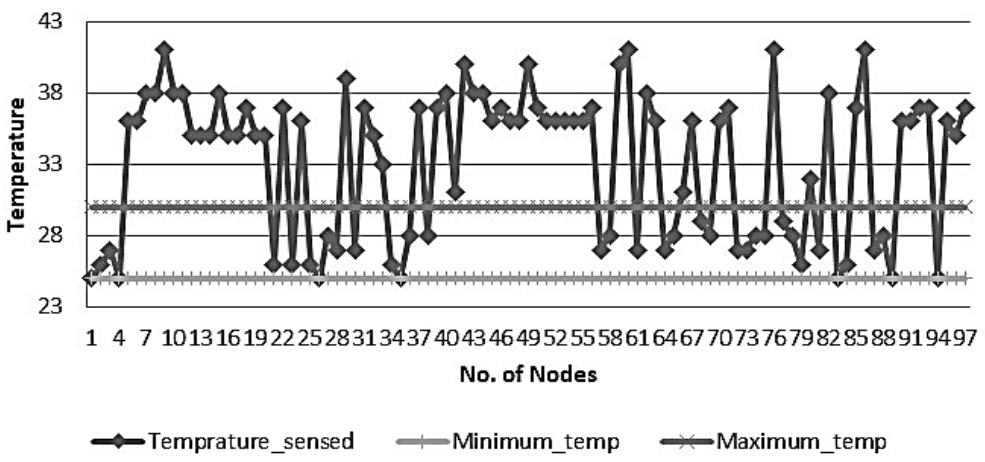

Figure 3. Time domain chart for temperature alert

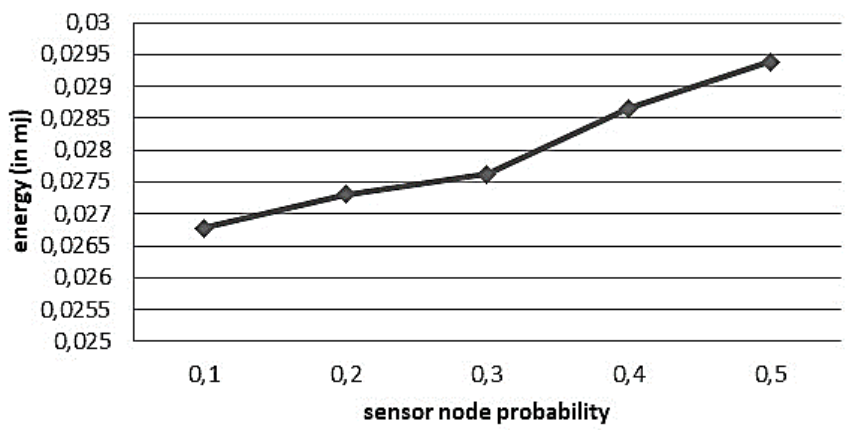

Figure 4. Average energy consumption

\subsection{Detection accuracy vs failure probability}

Fault detection rate is defined as the ratio among the number of faulty nodes which is identified correctly to the total number of wrongly sensed data; Figure 5 shows the fault detection accuracy rate by varying the node failure probability. Furthermore, performance comparison with existing model i.e., TPEFTED is depicted in same figure; moreover, in case of 0.5 sensor node failure probability, TPE-FTED achieves detection accuracy of 0.61. Similarly, in case of 0.4 and 0.3 , TPE-FTED achieves detection accuracy of 0.35 and 0.18 respectively; further, in case of 0.2 and 0.1 existing model i.e., TPE-FTED achieves detection accuracy of 0.038 and 0.18 respectively. However, in case of sensor node probability of 0.5 to 0.1 proposed model i.e., EDSM achieves absolute detection accuracy of 1.0.

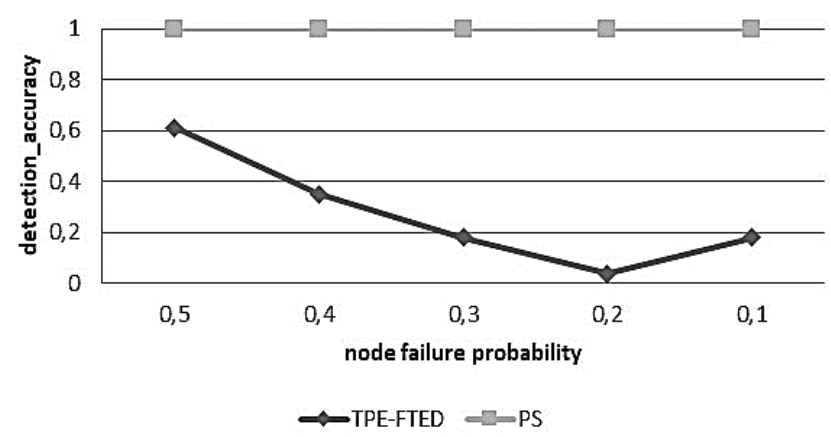

Figure 5. Fault detection rate versus sensor failure probability 


\subsection{False alarm rate vs sensor node failure probability}

False alarm rate is another parameter considered to evaluate the proposed model; in general, false alarm rate is computed through the mathematical ratio normal node (which is identified as the faulty nodes) to the absolute number of real nodes. Figure 6 shows the comparison of existing and proposed model over the various faulty node; Moreover, through the figure, it is observed that in case of 0.5 and 0.4 existing model gets the value of 0.81 and 0.96 respectively. Furthermore, in case of 0.3 and 0.2 , existing model gets false with value of 0.81 and 0.36 ; also, for 0.1 node fault probability, existing model gets 0.45 as false value. Moreover, less false alarm rate indicates better efficiency of model; thus, proposed model i.e., EDSM achieves false alarm rate of nearly 0 .

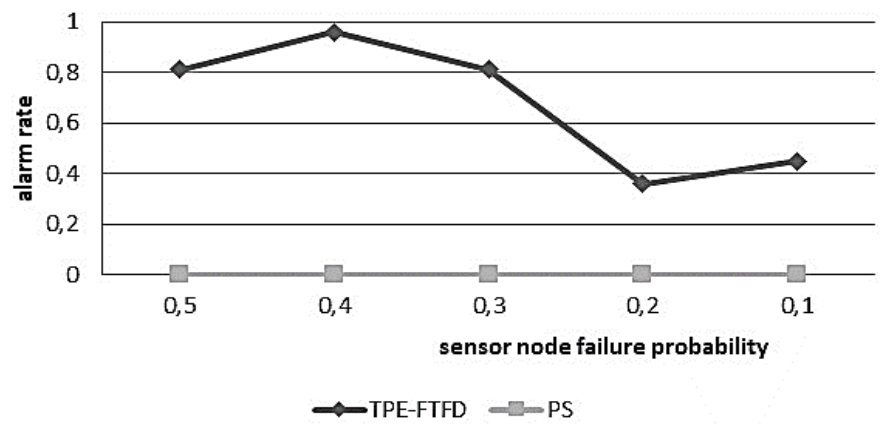

Figure 6. False alarm rate versus sensor failure probability

\subsection{Event detection accuracy versus sensor node failure probability}

Event detection accuracy is formulated as the ratio among the correctly identified nodes in occurred event to the total number of actual events; higher number of event detection accuracy indicates the model efficiency. Figure 7 shows the comparison of TPE-FTD and proposed model; in case of 0.5 and 0.4 existing model achieves detection accuracy of 0.68 ; however, proposed model achieves detection accuracy of 0.96 and 0.95 respectively. Similarly in case of 0.3 and 0.2 , existing model achieves the detection accuracy of 0.4 and 0 whereas proposed model achieves 1 and 0.9 respectively; at last, in case of 0.1 false node probability existing model achieves detection accuracy of 0 and 0.4 whereas proposed model achieves 0.9 and 1 .

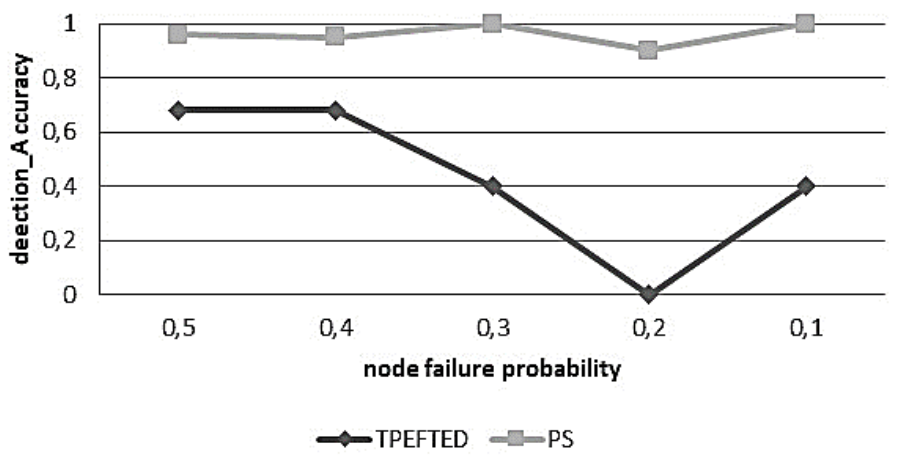

Figure 7. Event detection accuracy

\section{EVENT FALSE ALARM RATE VERSUS SENSOR FAILURE PROBABILITY}

Event false alarm rate is computed as the ratio among number of nodes considered as event node although it is faulty; Figure 8 shows the comparison of existing and proposed model considering the various sensor node failure probability; less false alarm rate indicates the model efficiency. Moreover, in case of 0.5 and 0.4 sensor node fault probability existing model gets 0.56 and 0.65 false alarm rate whereas proposed model gets 0.05 and 0.04 respectively. Similarly, in case of 0.3 and 0.2 fault probability existing model gets false alarm rate of 0.6 and 1 whereas in comparison proposed model i.e., EDSM achieves 0 and 0.1 . At last, in case of 0.1 sensor node fault probability existing model gets 0.2 and proposed model gets 0 false alarm. 


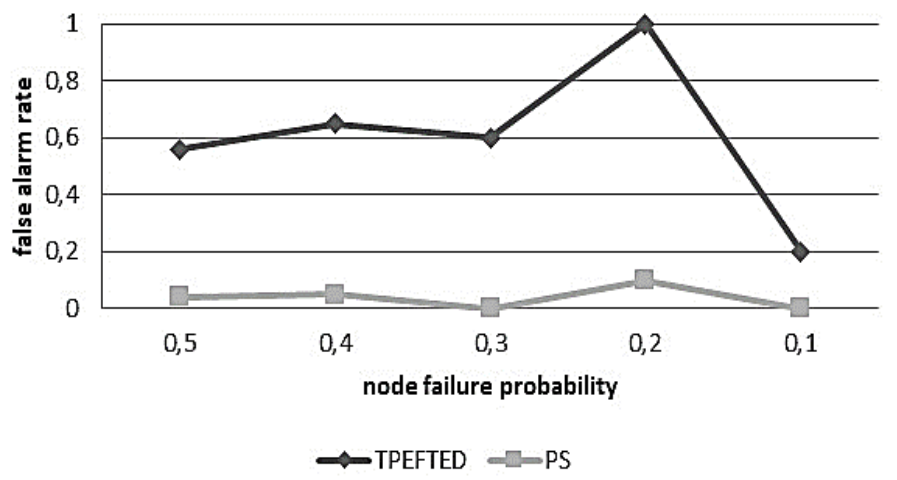

Figure 8. False alarm rate comparison

\section{COMPARATIVE ANALYSIS}

In order to prove the model efficiency of proposed model i.e., EDSM comparative analysis and further enhancement of proposed model is carried out in this section. Moreover, the comparison has been carried out considering four different cases i.e., fault detection rate, false alarm rate, event detection and event false alarm rate which is depicted in Figures 3-6 in respective manner. Furthermore, in case of fault detection rate proposed model achieves $48.44 \%$ improvisation for 0.5 sensor node failure probability and $96 \%$ improvisation is achieved for 0.4 sensor node failure probability; furthermore, in case of the 0.3 to 0.1 proposed model achieves the improvisation more than hundred percent as existing model accuracy gets lower further.

Similarly, considering false alarm rate, proposed model simply excels without having misidentified the faulty nodes i.e., with 0 false alarm rate in all four different node failure probabilities considered. Furthermore, considering the event detection rate, EDSM observes the improvisation of $41 \%$ in case of 0.5 probability $39.70 \%$ improvisation in 0.4 probabilities and 0.3 to 0.1 , it simply outperforms as the existing model fall flatly. In case of event false alarm rate, EDSM achieves improvisation of 92\% for 0.5 probabilities, $92.30 \%$ improvisation for 0.4 failure probability and $90 \%$ improvisation for 0.2 ; in case of 0.3 and 0.1 proposed model did not get single false alarm.

\section{CONCLUSION}

Farming is foundation of any national as well as harmonious society and for past several years' government has been focusing on the precision farming; moreover, there are various types of nuts produced in India, areca nut is one of the largest productivity in our country. Hence recent approach has been focused on precision farming of areca nut through smart sensing technology like wireless sensing technology incorporated with IoT; furthermore, this technology helps in analyzing the environmental factors like temperature, humidity as well as helps in monitoring the nutrients cycle like NPK. Hence, in this research work, we design and develop a sensing mechanism EDSM which aims at not only efficient sensing but monitoring and generating the alert; also energy consumption is consider as the primary factors for efficient sensing mechanism.

EDSM comprises various step which includes the mathematical model of energy consumption, efficient sensing, monitoring and packet size optimization. Furthermore, EDSM is evaluated considering the different parameter like detection accuracy, false alarm rate by comparing with the existing methodology; also throughout the evaluation it is observe that proposed model simply outperforms the existing model with significant optimization as in case various sensor node failure probability proposed model achieves $100 \%$ detection rate and does not get even a single false alarm rate. Although EDSM are proven to be efficient and solves various problem of existing model, there are still several areas like further optimal alert generation can be looked considering different parameter as precision farming possesses various factors.

\section{REFERENCES}

[1] Population Division of the United Nations Department of Economic and Social Affairs. World Population Projected to Reach 9.8 Billion in 2050, and 11.2 Billion in 2100. Accessed: Jul. 19, 2020. [Online]. Available: https://www.un.org/development/desa/en/news/population/world-population-prospects-2017.html

[2] Sustainable Agriculture Program | National Institute of Food and Agriculture. Accessed: Jul. 19, 2020. [Online]. Available: https://nifa. usda.gov/program/sustainable-agriculture-program 
[3] Soil Moisture Monitoring: A Selection Guide | Agriculture and Food. Accessed: Oct. 10, 2020. [Online]. Available: https://www.agric.wa. gov.au/horticulture/soil-moisture-monitoring-selection-guide

[4] M. B. de Morais Franca, F. J. O. Morais, P. Carvalhaes-Dias, L. C. Duarte, and J. A. S. Dias, "A multiprobe heat pulse sensor for soil moisture measurement based on PCB technology," IEEE Trans. Instrum. Meas., vol. 68, no. 2, pp. 606-613, Feb. 2019, doi: 10.1109/TIM.2018.2843605.

[5] G. Pandey, R. J. Weber, and R. Kumar, "Agricultural Cyber-Physical System: In-Situ Soil Moisture and Salinity Estimation by Dielectric Mixing," in IEEE Access, vol. 6, pp. 43179-43191, 2018, doi: 10.1109/ACCESS.2018.2862634.

[6] P. A. Neves, J. P. C. Rodrigues, M. Chen, and A. V. Vasilakos, "A multi-channel architecture for IPv6-enabled wireless sensor and actuator networks featuring PnP support," Journal of Network and Computer Applications, vol. 37, pp. 12-24, 2014, doi: 10.1016/j.jnca.2011.03.033.

[7] J. Lee and C. Teng, "An Enhanced Hierarchical Clustering Approach for Mobile Sensor Networks Using Fuzzy Inference Systems," in IEEE Internet of Things Journal, vol. 4, no. 4, pp. 1095-1103, Aug. 2017, doi: 10.1109/JIOT.2017.2711248

[8] H. E. Alami and A. Najid, "ECH: An Enhanced Clustering Hierarchy Approach to Maximize Lifetime of Wireless Sensor Networks," in IEEE Access, vol. 7, pp. 107142-107153, 2019, doi: 10.1109/ACCESS.2019.2933052.

[9] B. Kashyap and R. Kumar, "Sensing Methodologies in Agriculture for Soil Moisture and Nutrient Monitoring," in IEEE Access, vol. 9, pp. 14095-14121, 2021, doi: 10.1109/ACCESS.2021.3052478.

[10] H. Mahmoudzadeh, H. R. Matinfar, R. Taghizadeh-Mehrjardi, and R. Kerry, "Spatial prediction of soil organic carbon using machine learning techniques in Western Iran," Geoderma Regional, vol. 21, Jun. 2020, Art. no. e00260.

[11] W. R. Heinzelman, A. Chandrakasan, and H. Balakrishnan, "Energy ef_cient communication protocol for wireless microsensor networks," in Proc. 33rd Annu. Hawaii Int. Conf. Syst. Sci., p. 10, Jan. 2000.

[12] N. Israr and I. U. Awan, "Multihop clustering algorithm for load balancing in wireless sensor networks," Int. J. Simul., vol. 8, no. 3, pp. 13-24, 2007.

[13] B. Manzoor, N. Javaid, O. Rehman, M. Akbar, Q. Nadeem, A. Iqbal, and M. Ishfaq, "Q-LEACH: A new routing protocol for WSNs," ProcediaComput. Sci., vol. 19, pp. 9260-931, 2013.

[14] E. László, K. Tornai, G. Treplán, and J. Levendovszky, "Novel load balancing scheduling algorithms for wireless sensor networks," in Proc.4th Int. Conf. Commun. Theory, Rel., Qual. Service, Budapest, 2011, pp. 49-54.

[15] C. Gherbi, Z. Aliouat, and M. Benmohammed, "A novel load balancing scheduling algorithm for wireless sensor networks," $J$. Netw. Syst. Manage., vol. 27, no. 2, pp. 430-462, Apr. 2019, doi: 10.1007/s10922-018-9473-0.

[16] S. Lindsey and C. S. Raghavendra, "PEGASIS: Power-ef_cient gathering in sensor information systems," in Proc. IEEE Proc. Aerosp. Conf., vol. 3, Mar. 2002, p. 1-3, doi: 10.1109/AERO.2002.1035242.

[17] A. Singh, K. Babbar, and A. Malik, "Clustering and energy ef_cient routing protocol for wireless sensor network using genetic algorithm," Int. J. Comput. Appl., vol. 119, no. 7, pp. 40-43, Jun. 2015, doi: 10.5120/21083-3766.

[18] H. Ali, W. Shahzad, and F. A. Khan, "Energy-ef_cient clustering in mobile ad-hoc networks using multi-objective particle swarm optimization," Appl. Soft Comput., vol. 12, no. 7, pp. 1913-1928, Jul. 2012, doi: 10.1016/j.asoc.2011.05.036.

[19] F.-E. Bai, H.-H. Mou, and J. Sun, "Power-ef_cient zoning clustering algorithm for wireless sensor networks," in Proc. Int. Conf. Inf. Eng.Comput. Sci., Dec. 2009, pp. 1-4, doi: 10.1109/ICIECS.2009.5362734.

[20] K. Ahmed and M. Gregory, "Integrating wireless sensor networks with cloud computing," in Proc. 7th Int. Conf. Mobile Ad-Hoc Sensor Netw., Dec. 2011, pp. 364-366, doi: 10.1109/MSN.2011.86

[21] X. Meng, X. Shi, Z. Wang, S. Wu, and C. Li, "A grid-based reliable routing protocol for wireless sensor networks with randomly distributed clusters," Ad Hoc Netw., vol. 51, pp. 47-61, Nov. 2016, doi: 10.1016/j.adhoc.2016.08.004.

[22] T. J. Saleem, "A detailed study of routing in Internet of Things," Int. J.Eng. Sci. Innov. Technol., vol. 5, no. 3, May 2016.

[23] I. F. Akyildiz, W. Su, Y. Sankarasubramaniam, and E. Cayirci, "A survey on sensor networks," IEEE Commun. Mag., vol. 40, no. 8, pp. 102-114, Aug. 2002, doi: 10.1109/MCOM.2002.1024422.

[24] S. Yadav and R. S. Yadav, "A review on energy ef_cient protocols in wireless sensor networks," Wireless Netw., vol. 22, no. 1, pp. 335-350, Jan. 2016, doi: 10.1007/s11276-015-1025-x.

[25] S. Khan, A.-S. K. Pathan, and N. A. Alrajeh, Wireless Sensor Networks: Current Status and Future Trends. Boca Raton, FL, USA: CRC Press, Nov. 2016.

[26] H. El Alami and A. Najid, "Optimization of energy ef_ciency in wireless sensor networks and Internet of Things: A review of related works," in Nature-Inspired Computing Applications in Advanced CommunicationNetworks, ed. Hershey, PA, USA: IGI Global, 2020, pp. 89-127, doi: 10.4018/978-1-7998-1626-3.ch005.

[27] V. P. Kour and S. Arora, "Recent Developments of the Internet of Things in Agriculture: A Survey," in IEEE Access, vol. 8, pp. 129924-129957, 2020, doi: 10.1109/ACCESS.2020.3009298.

[28] R. Wan, N. Xiong, and N. T. Loc, "An energy-efficient sleep scheduling mechanism with similarity measure for wireless sensor networks," Hum.- Centric Comput. Inf. Sci., vol. 8, no. 1, p. 18, Dec. 2018, doi: 10.1186/s13673-018-0141-x.

[29] H. Wang, Y. Li, T. Chang, and S. Chang, "An effective scheduling algorithm for coverage control in underwater acoustic sensor network," Sensors, vol. 18, no. 8, p. 2512, Aug. 2018, doi: 10.3390/s18082512.

[30] M. Song, W. Lu, H. Peng, Z. Xu, and J. Hua, "A method of balanced sleep scheduling in renewable wireless sensor networks," in Proc. Int. Conf. Mach. Learn. Intell. Commun., 2018, pp. 293-302, doi: 10.1007/978-3-030-00557-3 30

[31] D. Xue and W. Huang, "Smart Agriculture Wireless Sensor Routing Protocol and Node Location Algorithm Based on Internet of Things Technology," in IEEE Sensors Journal, 2020, doi: 10.1109/JSEN.2020.3035651.

[32] Z. Zhang, S. Glaser, T. Watteyne, and S. Malek, "Long-term Monitoring of the Sierra Nevada Snowpack Using Wireless Sensor Networks," in IEEE Internet of Things Journal, 2020, doi: 10.1109/JIOT.2020.2970596.

[33] S. K. Roy, S. Misra, N. S. Raghuwanshi, and S. K. Das, "AgriSens: IoT-Based Dynamic Irrigation Scheduling System for Water Management of Irrigated Crops," in IEEE Internet of Things Journal, vol. 8, no. 6, pp. 5023-5030, 2021, doi: 10.1109/JIOT.2020.3036126.

[34] L. Liu, G. Han, Y. He, and J. Jiang, "Fault-Tolerant Event Region Detection on Trajectory Pattern Extraction for Industrial Wireless Sensor Networks," in IEEE Transactions on Industrial Informatics, vol. 16, no. 3, pp. 2072-2080, March 2020, doi: 10.1109/TII.2019.2933238. 


\section{BIOGRAPHIES OF AUTHORS}
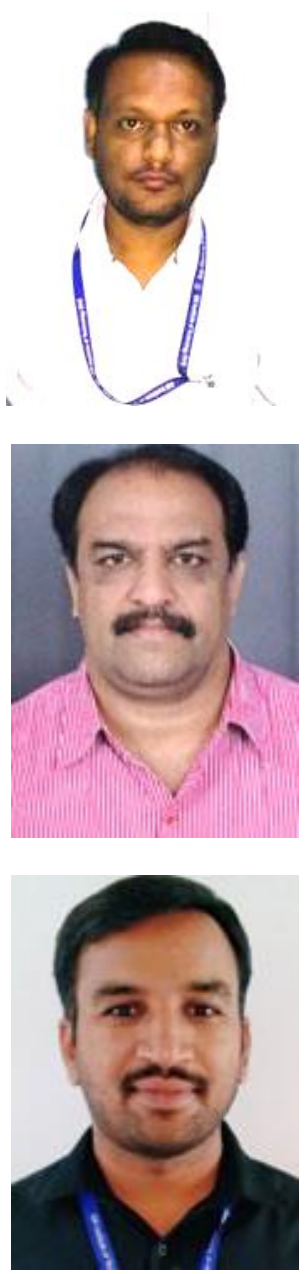

Niranjan Murthy Chandrashekarappa (iD SIS SC P Assistant professor Department of Computer Science and Engineering I completed BE in VTU university and received M.Tech in Kuvempu University Gate qualified In 2008 total 10 year teaching experience I guided project emotional based music theory and mobile application of RTO system my interesting subject are automata theory and compiler design I am doing research in cloud computing and IOT Research Scholar Vtu, Belgaum Assistant professor, Department of CSE, GMIT Davanagere, Karnataka. He can be contacted at email: nirucs190@gmail.com.

SanjayPande Mysore Bhagwan (D) If] SC P Professor, Department of Computer Science \& Engineering. G M Institute of Technology, Davangere. University: Visvesvaraya Technological University "JnanaSangama” Belagavi-590018, completed Phd An algorithmic model for exploratory analysis of trace elements in cognition and recognition of neurological disorders. Member of Eurasia Research-membership ID: TERA-M19624, Membership Category: Lifetime, Association: Teaching and Education Research Association (TERA). He can be contacted at email: rakroop99@gmail.com.

Kotreshi Shivabasappa Nagur ${ }^{D}$ SC $\mathrm{P}$ Studied his B.E degree in UBDTCE and M.Tech degree in BIET, Davangere. His areas of interest are IoT, Web Technologies, Data Science, Image Processing and Machine learning. He guided nearly 20 UG/PG projects in these areas. He has 16 years teaching experience and currently he is working as Asst. Professor in the Dept. of CSE, GMIT, Davangere, and Karnataka. He can be contacted at email: kotreshisn@gmail.com. 